\title{
Efecto de microorganismos con potencial probiótico en la calidad del agua y el crecimiento de camarón Litopenaeus vannamei (Decapoda: Penaeidae) en cultivo intensivo
}

\author{
Carolina Esther Melgar Valdes ${ }^{1,2 *}$, Everardo Barba Macías ${ }^{1}$, Carlos Alfonso Álvarez-González ${ }^{3}$, \\ Cristian Tovilla Hernández ${ }^{4} \&$ Alberto J. Sánchez ${ }^{5}$ \\ 1. El Colegio de la Frontera Sur, Unidad Villahermosa, Depto. de Aprovechamiento y Manejo de Recursos Acuáticos. \\ Carretera Villahermosa-Reforma km. 15.5, Ranchería Guineo 2 2a sección C.P. 86280 Villahermosa, Tabasco, México; \\ cemv81@gmail.com, ebarba@ecosur.mx \\ 2. Universidad Juárez Autónoma de Tabasco. División Académica Multidisciplinaria de los Ríos, Carretera Tenosique- \\ Estapilla km 1 s/n, C.P. 86901, Tenosique, Tabasco, México; carolina.melgar@ujat.mx \\ 3. Laboratorio de Acuicultura Tropical, DACBIOL-UJAT, Carretera Villahermosa-Cárdenas km. $0.5 \mathrm{~s} / \mathrm{n}$, C.P. 86039, \\ Villahermosa, Tabasco, México; alfonso.alvarez@dacbiol.ujat.mx \\ 4. El Colegio de la Frontera Sur, Unidad Tapachula. Carretera Antiguo Aeropuerto km. 2.5, C.P. 30700, Tapachula, \\ Chiapas, México; ctovilla@ecosur.mx \\ 5. Laboratorio de Hidrobiología, DACBIOL-UJAT, Carretera Villahermosa-Cárdenas km. $0.5 \mathrm{~s} / \mathrm{n}$, C.P. 86039, \\ Villahermosa, Tabasco, México; alberthoj.sanchez@gmail.com \\ * Correspondencia
}

Recibido 26-VI-2012. Corregido 14-X-2012. Aceptado 08-XI-2012.

\begin{abstract}
Microorganisms effect with probiotic potential in water quality and growth of the shrimp Litopenaeus vannamei (Decapoda: Penaeidae) in intensive culture. The use of probiotics has gained acceptance in aquaculture, particularly in maintaining water quality and enhancing growth in organisms. This study analyzed the effect of the commercial (EM ${ }^{\mathrm{TM}}$, Japan) natural product composed by (Rhodopseudomonas palustris, Lactobacillus plantarum, Lactobacillus casei and Saccharomyces cerevisiae) added to the water, in order to determine its effect in water quality, sediment and growth of $L$. vannamei under intensive culture. The evaluation included three treatments with a weekly addition of EM: i) tanks without probiotics (C), ii) tanks with a dose of $4 \mathrm{~L} / \mathrm{ha}$ (EM1) and iii) tanks with a dose of 10L/ha (EM2). The treatment $\mathrm{C}$ was carried out three times, while treatments EM1 and EM2 were carried out four times. A total of 4350 shrimps were measured for total length and weight, to calculate total and porcentual weight gain, daily weight gain, specific growth rate (TCE), and food conversion factor (FCA); besides, the survival rate was estimated. The use of probiotics allowed a shorter harvest time in treatments EM1 (90d) and EM2 (105d) with relation to the treatment C (120d). Treatments EM1 and EM2 were within the recommended intervals for culture, with respect to treatment $\mathrm{C}$. The use of probiotic

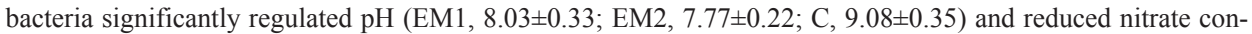
centration $(\mathrm{EM} 1,0.64 \pm 0.25 \mathrm{mg} / \mathrm{L} ; \mathrm{EM} 2,0.39 \pm 0.26 \mathrm{mg} / \mathrm{L} ; \mathrm{C}, 0.71 \mathrm{mg} / \mathrm{L})$. Water $\mathrm{pH}$ mostly explained the variance with respect to the treatments. Treatment EM2 presented the greatest removal of organic matter $(1.77 \pm 0.45 \%)$, whereas the contents of extractable phosphorus increased significantly in treatment EM1 with $21.6 \pm 7.99 \mathrm{mg} / \mathrm{kg}$ and in treatment EM2 with $21.6 \pm 8.45 \mathrm{mg} / \mathrm{kg}$ with control relation (14.3 \pm 5.47$)$. The shrimp growth was influenced by dissolved oxygen, salinity and $\mathrm{pH}$ in the sediment, establishing that salinity was the most important variable in the weight with a negative association. Treatment EM1 recorded an improved TCE $(2.69 \pm 0.35 \% / \mathrm{d})$ and FCA $(1.46 \pm 0.20$ ) with relation to the control treatment (TCE, $1.88 \pm 0.25 \% / \mathrm{d}$; FCA, $2.13 \pm 0.48$ ). Survival was significantly greater in treatments containing probiotics with $61 \pm 8.76 \%$ and $60 \pm 10.5 \%$ for EM1 and EM2, respectively. This study indicated the positive effect obtained with the use of this commercial probiotic, to improve culture conditions and growth parameters in an intensive culture of $L$. vannamei. Rev. Biol. Trop. 61 (3): 1215-1228. Epub 2013 September 01.
\end{abstract}

Key words: aquaculture, growth, Litopenaeus vannamei, probiotic, water quality. 
La camaronicultura se ha caracterizado por tener un acelerado crecimiento y una rápida expansión económica, circunstancia que ha incidido en la intensificación de los sistemas de producción (Ajitha et al. 2004). Sin embargo, durante los últimos 20 años los productores de camarón han sufrido enormes pérdidas económicas, debido al incremento de enfermedades que afectan su producción y exportación (Zokaei et al. 2009, Panwichian et al. 2010); además, porque han encontrado su mayor dificultad en el manejo de la calidad del agua, causada por la acumulación de materia orgánica y metabolitos tóxicos para los camarones, como los compuestos nitrogenados (Ladino-Orjuela \& Rodríguez-Pulido 2009, Zhou et al. 2009).

No obstante, el uso de agentes químicos (antibióticos, terapéuticos) se presentó como la mejor opción para prevenir y controlar los problemas relacionados con enfermedades en el cultivo de camarón (Qi et al. 2009). Sin embargo, los efectos adversos ocasionados por la resistencia de las bacterias patógenas y sus repercusiones en la salud humana, originaron la búsqueda de nuevas tecnologías que permitieran disminuir: i) la perdida potencial de los productores, ii) el riesgo en el consumo, y iii) su impacto en el ambiente (Zokaei et al. 2009).

Diversos autores han propuesto la implementación de tecnologías limpias a través del uso de probióticos en la acuicultura, los cuales han sido definidos como "microorganismos con efectos benéficos sobre el hospedero por la modificación del ambiente huésped-hospedero o la modificación de su comunidad microbiana, por la mejora en la asimilación de alimento o de su valor nutricional, por mejoramiento de la respuesta del hospedero ante enfermedades o por la mejora en la calidad de su medio ambiente" (Vershuere et al. 2000). Estos consorcios microbianos también han sido denominados como microorganismos eficientes.

Diferentes investigaciones han demostrado que los microorganismos benéficos pueden: 1) incrementar el valor nutricional (Venkat et al. 2004, Balcázar et al. 2007, Banerjee et al. 2010), 2) aumentar la supervivencia y disminuir enfermedades mediante la inhibición del crecimiento de bacterias patógenas (Sansawat \& Thirabunyanon 2009, Ismail \& Soliman 2010), 3) mantener y mejorar la calidad del agua con la reducción de concentraciones de amonio, nitrito y nitrato en el agua (Shariff et al. 2001, Jana \& Jana 2003, Gutierrez-Wing \& Malone 2006, Chae-Woo et al. 2009) y 4) disminuir la carga elevada de materia orgánica (Douilett 1998, Dalmin et al. 2001). En contraste, existen estudios que han reportado efectos nulos o negativos con el uso de probióticos, como infecciones cutáneas o aumento en la mortalidad (McIntosh et al. 2000, Wang et al. 2000, Günther \& Jiménez-Montealegre 2004), con lo cual se ha evidenciado que el uso de los probióticos no siempre son benéficos, debido a que dependen de diversos factores como son: las especies de cultivo, los sistemas de producción, la escala de cultivo (laboratorio y granjas), la densidad de siembra, utilización de microorganismos provenientes de otros ambientes y la dosis de los microorganismos (Verschuere et al. 2000, Panwichian et al. 2010).

Por otro lado, la eficacia de los probióticos comerciales para el cultivo de camarón ha sido severamente cuestionada y criticada (Villamil \& Martínez-Silva 2009); sin embargo, estos productos han sido utilizados con mayor frecuencia por los productores debido a que pueden encontrarse con mayor disponibilidad en el mercado, a diferencia de los microorganismos aislados y evaluados por comunidades científicas, los cuales actualmente son poco usados.

El camarón blanco Litopenaeus vannamei (Boone 1931) es una especie de gran importancia debido a su gran posibilidad de manejo en diferentes sistemas de cultivo, capacidad de adaptación a intervalos razonables a variaciones ambientales, alta tasa de supervivencia y rápido crecimiento, pero principalmente, por el establecimiento de un buen precio en el mercado internacional (Barón-Sevilla et al. 2004, Valdez et al. 2008).

El objetivo de este trabajo fue analizar el efecto de una mezcla comercial de microorganismos con potencial probiótico en la calidad del agua y sedimento, así como en el crecimiento de 
postlarvas de L. vannamei en un sistema de cultivo intensivo en Tabasco, México.

\section{MATERIALES Y MÉTODOS}

Obtención y cultivo de microorganismos: Se utilizó un probiótico comercial (Tecnología $\mathrm{EM}^{\mathrm{TM}}$, Japón), el cual se encuentra distribuido en la mayor parte del mundo. El producto contiene tres tipos de microorganismos eficientes (EM) en estado de latencia: bacterias fotosintéticas (Rhodopseudomonas palustris con $2 \times 10^{3} \mathrm{UFC} / \mathrm{mL}$ ), bacterias lácticas (Lactobacillus plantarum y Lactobacillus casei con $5 \times 10^{4} \mathrm{UFC} / \mathrm{mL}$, respectivamente) y levaduras (Saccharomyces cerevisiae con $4 \times 10^{3} \mathrm{UFC} /$ $\mathrm{mL}$ ). Los microorganismos fueron inoculados en un sustrato a base de melaza comercial y agua para su activación, manteniéndose en fermentación durante siete días a una temperatura entre 36.5 y $37^{\circ} \mathrm{C}$ según la metodología sugerida por los fabricantes (Anónimo 2008).

Granja productora de camarón: El estudio se realizó en una granja comercial de cultivo intensivo, ubicada en la zona costera del municipio de Cárdenas (18²0'54" N 9332'40" W), Tabasco, México. El trabajo en campo inició en marzo y terminó en junio 2008. El experimento se llevó a cabo en estanques individuales de tierra (sustrato arcilloso) de 3ha, considerándose un ciclo de cultivo de 120 días. Las postlarvas (PL) se obtuvieron de un laboratorio certificado provenientes de un mismo lote de reproductores con pesos $(0.002 \mathrm{~g})$ y tallas homogéneas $(12 \mathrm{~mm})$. El proceso de siembra fue simultáneo con una densidad de $45 \mathrm{PL} / \mathrm{m}^{2}$. El manejo del sistema de cultivo se siguió conforme al programa operacional de los técnicos de la granja.

Evaluación del probiótico en el cultivo de camarón: El diseño experimental consistió en el suministro de diferentes dosis del probiótico comercial adicionado en el agua durante el ciclo de cultivo. La mezcla microbiana se agregó semanalmente y después de cada recambio de agua (drenando primero la cantidad apropiada de agua entre 5-10\% y luego rellenándolo mediante bombeo) en los estanques, esta fue de la siguiente manera: tratamiento $1(\mathrm{C})$, estaques sin dosificación del producto (control), tratamiento 2 (EM1), estanques adicionados con una dosis de $4 \mathrm{~L} / \mathrm{ha}$, y tratamiento 3 (EM2), estanques adicionados con dosis de 10L/ha. El tratamiento C se replicó tres veces, mientras que los tratamientos EM1 y EM2 se replicaron cuatro veces. La alimentación se realizó desde la etapa de siembra hasta la talla de comercialización (120d) con base al programa de alimentación para camarón blanco (API-Camarón, Malta Clayton, EE.UU). La tasa de alimentación fue ajustada semanalmente por los técnicos de la granja de acuerdo con las estimaciones del incremento en peso corporal (proteína: postlarva $(3-4 \mathrm{~g})=40 \%$, postlarva $(4-8 \mathrm{~g})=35 \%$, juvenil $(8-15 \mathrm{~g})=30 \%)$, tasa de supervivencia y la cantidad de alimento remanente en los comedores dos horas después de cada alimentación, obteniéndose cuatro dosis diarias de alimentación en la etapa de postlarva $\mathrm{y}$ tres dosis diarias en la etapa de juvenil.

Medición de los parámetros ambientales: La calidad del agua fue monitoreada cada dos semanas durante el día, entre las 7:00 y 9:00hr. Se determinaron los parámetros de $\mathrm{pH}$, temperatura, oxígeno disuelto y salinidad con un equipo Hanna HI 95928 (EEUU) a una profundidad de $50 \mathrm{~cm}$. Las concentraciones de nitrógeno amoniacal total (NAT) y nitrato $\left(\mathrm{NO}_{3}-\mathrm{N}\right)$ fueron estimadas con un medidor de amonio, Hanna HI 9828 (EEUU). La transparencia y la profundidad del agua se midieron in situ con el disco de Secchi. Al mismo tiempo, el sedimento se recolectó a $15 \mathrm{~cm}$ de profundidad con un nucleador de PVC (Kristensen et al. 1995). Posteriormente, las muestras fueron trasladadas al laboratorio donde se determinaron las variables de $\mathrm{pH}$, materia orgánica, nitrógeno total y fósforo extraíble de acuerdo a los métodos provistos por la NOM-021-SEMARNAT-2000.

Medición de los parámetros de crecimiento: Cada 14 días durante el ciclo de 
cultivo se capturaron aleatoriamente 50 camarones, a los cuales se les midió el peso $(\mathrm{g})$ con una balanza electrónica (Ohaus $\pm 0.001 \mathrm{~g}$ ), y la longitud total $(\mathrm{mm})$ con un calibrador electrónico (Truper CALDI-6MP $\pm 0.01 \mathrm{~mm}$ ). La muestra total de camarones pesados y medidos fue 4 350 [organismos medidos $\mathrm{x}$ número de réplicas por tratamiento $\mathrm{x}$ (días de cultivo/frecuencia de medición)]. Los parámetros de crecimiento fueron calculados conforme a lo reportado por Zokaei et al. (2009), quienes incluyeron: ganancia en peso $(\mathrm{g} /$ camarón $)=$ Peso final $(\mathrm{g})$ peso inicial $(\mathrm{g})$, ganancia en peso $(\%)=\mathrm{Peso}$ final $(\mathrm{g})$-peso inicial $(\mathrm{g}) /$ peso inicial $\mathrm{x} 100$, peso diario ganado $(\mathrm{g} / \mathrm{d})=$ Peso final $(\mathrm{g}) \times$ peso inicial/días de cultivo, tasa de crecimiento específica $(\mathrm{TCE}, \% / \mathrm{d})=$ ln Peso final-ln peso inicial/ días de cultivo x 100, factor de conversión alimenticia $($ FCA $)=$ Alimento seco entregado/peso húmedo ganado. El porcentaje de supervivencia se determinó al final del experimento como la relación entre el número final y el número inicial de los camarones en cultivo. Esta tasa se expresa como el porcentaje de supervivencia, según la siguiente fórmula (Saad et al. 2009): Supervivencia $(\%)=$ (Número final de camarones/Número inicial de camarones) x 100 .

A los parámetros ambientales, de crecimiento y supervivencia de los camarones se les aplicó un análisis de varianza (ANDEVA) de una vía. Los valores de $\mathrm{p}<0.05$ fueron considerados significativamente diferentes. Cuando mostraron diferencias significativas entre las medias de los tratamientos, se utilizó un análisis a posteriori y la prueba de Tukey (HSD) para identificar la naturaleza de estas diferencias $(p<0.05)$ (Daniel 2008). Se realizó un Análisis Múltiple Discriminante (AMD) para determinar las variables que contribuyeron en el comportamiento de los parámetros ambientales en relación a los tratamientos (Zar 2010). Asimismo, se aplicó un Análisis de Correspondencia Canónica (ACC) para determinar las asociaciones entre los parámetros de crecimiento (peso y talla) y los parámetros ambientales resultantes del AMD (Ter Braak \& Verdonshot 1995). Todos los análisis estadísticos se realizaron con el paquete STATISTICA versión 7.0 para Windows (StatSoft 2004).

\section{RESULTADOS}

Parámetros ambientales en el cultivo de camarón: En el cuadro 1 se presentan los parámetros fisicoquímicos obtenidos en el agua y sedimento de los estanques durante el cultivo de camarón. La temperatura, salinidad y $\mathrm{pH}$ fueron significativamente diferentes (Tukey, $\mathrm{p}<0.05$ ) en los tratamientos C, EM1 y EM2 $(F=57.16, p<0.05)$. Sin embargo, el tratamiento $\mathrm{C}$ presentó el mayor valor promedio en temperatura $\left(32.4 \pm 2.65^{\circ} \mathrm{C}\right)$ y $\mathrm{pH}(9.08 \pm 0.35)$. La menor concentración de oxígeno disuelto se observó en el tratamiento C $(4.38 \pm 2.05 \mathrm{mg} / \mathrm{L})$, el cual mostró diferencias significativas (Tukey, $\mathrm{p}<0.05$ ) respecto a los tratamientos EM1 y EM2. Con relación a las concentraciones de $\mathrm{NO}_{3}-\mathrm{N}$ y NAT, el tratamiento EM2 fue significativamente diferente (Tukey, $\mathrm{p}<0.05$ ) $(0.39 \pm 0.26 \mathrm{mg} / \mathrm{L}, 0.30 \pm 0.15 \mathrm{mg} / \mathrm{L})$ en comparación con los tratamientos EM1 $(0.64 \pm 0.25 \mathrm{mg} / \mathrm{L}$, $0.36 \pm 0.22 \mathrm{mg} / \mathrm{L}$ ) y el tratamiento $\mathrm{C}$, el cual registró los valores máximos de $\mathrm{NO}_{3}-\mathrm{N}$ y NAT $(0.71 \pm 0.22 \mathrm{mg} / 1,0.36 \pm 0.18 \mathrm{mg} / \mathrm{L})$. En los tratamientos EM1 y EM2 no se observaron diferencias significativas (Tukey, $p>0.05$ ) en la transparencia y la profundidad. Caso contrario, para el tratamiento $\mathrm{C}$ donde fue significativamente diferente (Tukey, $\mathrm{p}<0.05$ ) en estas variables $(8.79 \pm 8.11 \mathrm{~cm}, 56.4 \pm 19.7 \mathrm{~cm})$ y en el cual se presentaron los valores mínimos.

En relación a los parámetros fisicoquímicos en sedimento (Cuadro 1), los tratamientos C, EM1 y EM2 fueron significativamente diferentes (Tukey, $\mathrm{p}<0.05$ ) en materia orgánica y $\mathrm{pH}$. Los mayores valores estuvieron en los tratamientos C (2.64 $\pm 0.84 \%)$ para materia orgánica y EM2 para el pH (7.99 \pm 0.36$)$. Los contenidos de fósforo extraíble no registraron diferencias significativas (Tukey, $\mathrm{p}>0.05$ ) entre los tratamientos EM1 y EM2. Sin embargo, ambos tratamientos mostraron los mayores valores significativamente $(\mathrm{p}<0.05)(21.6 \pm 7.99 \mathrm{mg} / \mathrm{kg}$, $21.6 \pm 8.45 \mathrm{mg} / \mathrm{kg}$ ) en relación al tratamiento C $(14.3 \pm 5.47 \mathrm{mg} / \mathrm{kg})$. En el tratamiento EM1 
CUADRO 1

Parámetros ambientales de los estanques control y tratamiento durante el cultivo de camarón

TABLE 1

Environmental parameters in treatment and control ponds during shrimp culture

\begin{tabular}{|c|c|c|c|}
\hline \multirow{2}{*}{ Parámetros } & \multicolumn{3}{|c|}{ Tratamiento } \\
\hline & $\mathrm{C}$ & EM1 & EM2 \\
\hline \multicolumn{4}{|l|}{ Agua } \\
\hline Oxígeno disuelto (mg/L) & $4.38 \pm 2.05^{\mathrm{a}}$ & $5.29 \pm 1.37^{\mathrm{b}}$ & $5.05 \pm 1.18^{\mathrm{b}}$ \\
\hline Temperatura $\left({ }^{\circ} \mathrm{C}\right)$ & $32.4 \pm 2.65^{\mathrm{a}}$ & $30.5 \pm 1.57^{b}$ & $28.9 \pm 2.67^{\mathrm{c}}$ \\
\hline Salinidad (ups) & $19.8 \pm 4.00^{\mathrm{a}}$ & $25.8 \pm 3.87^{\mathrm{b}}$ & $14.4 \pm 7.14^{\mathrm{c}}$ \\
\hline $\mathrm{pH}$ & $9.08 \pm 0.35^{\mathrm{a}}$ & $8.03 \pm 0.33^{b}$ & $7.73 \pm 0.22^{\mathrm{c}}$ \\
\hline $\mathrm{NO}_{3}-\mathrm{N}(\mathrm{mg} / \mathrm{L})$ & $0.71 \pm 0.22^{\mathrm{a}}$ & $0.64 \pm 0.25^{\mathrm{a}}$ & $0.39 \pm 0.26^{b}$ \\
\hline NAT (mg/L) & $0.36 \pm 0.18^{\mathrm{a}}$ & $0.36 \pm 0.22^{\mathrm{a}}$ & $0.30 \pm 0.15^{\mathrm{b}}$ \\
\hline Transparencia $(\mathrm{cm})$ & $8.79 \pm 8.11^{\mathrm{a}}$ & $16.3 \pm 9.65^{\mathrm{b}}$ & $14.7 \pm 5.77^{\mathrm{b}}$ \\
\hline Profundidad $(\mathrm{cm})$ & $56.4 \pm 19.7^{\mathrm{a}}$ & $74.2 \pm 17.2^{\mathrm{b}}$ & $75.4 \pm 16.4^{\mathrm{b}}$ \\
\hline \multicolumn{4}{|l|}{ Sedimento } \\
\hline $\mathrm{pH}$ & $7.75 \pm 0.33^{\mathrm{a}}$ & $7.47 \pm 0.41^{\mathrm{b}}$ & $7.99 \pm 0.36^{\mathrm{c}}$ \\
\hline Fósforo extraíble (mg/kg) & $14.3 \pm 5.47^{\mathrm{a}}$ & $21.6 \pm 7.99^{b}$ & $21.6 \pm 8.45^{\mathrm{b}}$ \\
\hline Nitrógeno total (\%) & $0.16 \pm 0.04^{\mathrm{a}}$ & $0.12 \pm 0.03^{\mathrm{a}}$ & $0.36 \pm 0.42^{\mathrm{b}}$ \\
\hline Materia orgánica (\%) & $2.64 \pm 0.84^{\mathrm{a}}$ & $2.01 \pm 0.75^{\mathrm{b}}$ & $1.77 \pm 0.45^{\mathrm{c}}$ \\
\hline $\mathrm{N}$ & 3 & 4 & 4 \\
\hline
\end{tabular}

Promedios en la misma fila con superíndices diferentes presentan diferencia significativa $(\mathrm{p}<0.05)$.

Promedio \pm desviación estándar.

C: Control. EM1: Probiótico dosis 4L/ha. EM2: Probiótico dosis 10L/ha.

se observó el mínimo valor de nitrógeno total $(0.12 \pm 0.03 \%)$, el cual no fue significativamente diferente $(p>0.05)$ con el tratamiento $C$, en comparación con el tratamiento EM2, donde se mostraron diferencias significativas (Tukey, $\mathrm{p}<0.05)(21.6 \pm 8.45 \%)$.

En relación al análisis múltiple discriminante aplicado a los parámetros ambientales en agua y sedimento, se observó que sólo seis parámetros fueron significativos en el modelo $\left(\lambda=0.067, \mathrm{~F}_{22,484}=63.244, \mathrm{p}<0.05, \mathrm{R}^{2}=0.915\right)$ : $\mathrm{pH}$ en agua $(\lambda=0.174, \mathrm{p}<0.05)$, salinidad $(\lambda=0.096, \mathrm{p}<0.05)$, oxígeno disuelto $(\lambda=0.084$, $\mathrm{p}<0.05)$, pH en sedimento $(\lambda=0.072, \mathrm{p}<0.05)$, fósforo extraíble $(\lambda=0.071, \mathrm{p}<0.05)$ y nitrógeno total en sedimento $(\lambda=0.071, \mathrm{p}<0.05)$. La distribución de las observaciones correspondientes en función del espacio discriminante entre la primera y segunda función se presenta en la figura 1. El mayor grado de traslape se presentó en los tratamientos EM1 y EM2, mientras que el menor grado de traslape lo presentaron el tratamiento C y EM1, con lo cual, se indicó que el 93\% de las variables canónicas se encontraron correctamente clasificadas. De acuerdo a los valores de los coeficientes estandarizados para las variables canónicas en la primer función discriminante, el efecto discriminante entre los tres tratamientos en el cultivo de camarón mostró que la variable $\mathrm{pH}$ en agua posee una carga canónica de $82 \%\left(\lambda_{\mathrm{p}}=0.382\right.$, $\mathrm{F}_{\mathrm{R}}=194, \mathrm{p}<0.05, \mathrm{~T}=79 \%, \mathrm{R}^{2}=20 \%$ ), seguida por fósforo extraíble con $19 \%\left(\lambda_{p}=0.944\right.$, $\mathrm{F}_{\mathrm{R}}=7.11, \mathrm{p}<0.05, \mathrm{~T}=89 \%, \mathrm{R}^{2}=10 \%$ ), cuyo efecto es inversamente proporcional. El resto de las variables no presentaron una carga canónica significativa.

Parámetros de crecimiento y supervivencia en el cultivo de camarón: Las variaciones obtenidas en el peso y longitud de los camarones en el período de evaluación en la granja se muestran en la figura 2. En base al ciclo de cultivo, se observó que el tratamiento 


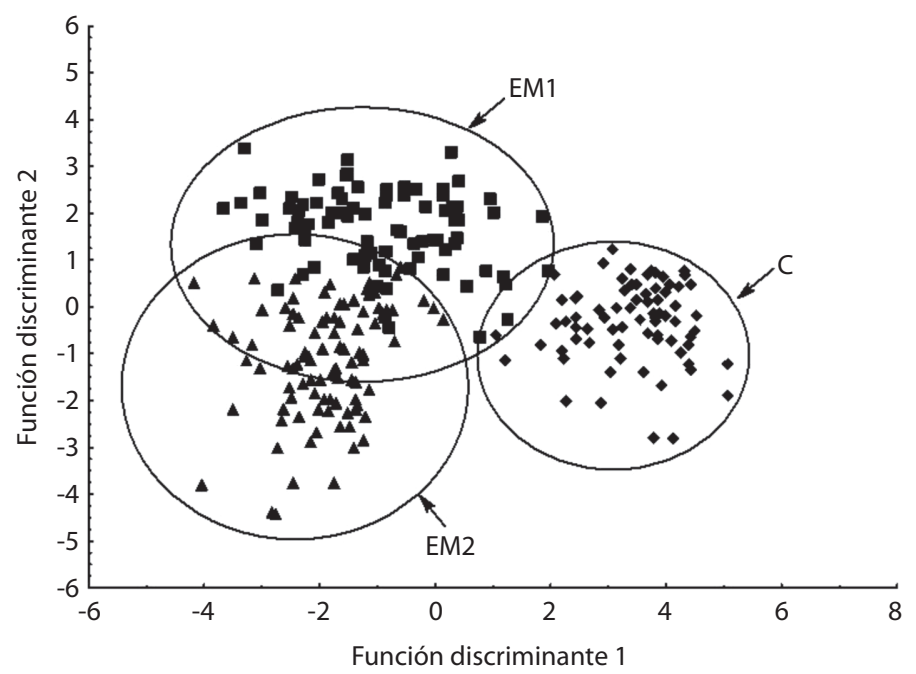

Fig. 1. Análisis discriminante de los parámetros ambientales que explican la variabilidad de los tratamientos (área de traslape: 95\% intervalo de confianza). C: Control. EM1: Probiótico dosis 4L/ha. EM2: Probiótico dosis 10L/ha.

Fig. 1. Discriminant analysis of environmental parameters which explain the treatments variability (overlap area: confidence interval 95\%). C: Control. EM1: Doses probiotic 4L/ha. EM2: Doses probiotic 10L/ha.

EM1 presentó el menor tiempo de cosecha con 90d, seguido del tratamiento EM2 con 105d y finalmente, el tratamiento $\mathrm{C}$ con $120 \mathrm{~d}$. En relación al peso y longitud total entre los tratamientos existieron diferencias significativas $(\mathrm{F}=29.7, \mathrm{p}<0.05)$. El peso y longitud total al final del cultivo (talla de cosecha) de los camarones mostraron que el tratamiento EM1 fue de $11.7 \pm 8.00 \mathrm{~g}$ y $114 \pm 12.6 \mathrm{~mm}$, mientras que en el tratamiento EM2 fue de $8.06 \pm 1.72 \mathrm{~g}$ y $96.3 \pm 8.17 \mathrm{~mm}$, y en el tratamiento $\mathrm{C}$ fue de $8.02 \pm 1.24 \mathrm{~g}$ y $97.4 \pm 6.60 \mathrm{~mm}$, respectivamente. El tratamiento EM1 registró los mayores valores en el peso y longitud total al final del cultivo, observándose diferencias significativas (Tukey, $\mathrm{p}<0.05$ ) entre los camarones tratados con EM2 y C. Sin embargo, se identificó que el tratamiento EM2 fue ligeramente mayor en el peso final respecto al tratamiento $\mathrm{C}$.

Los parámetros de crecimiento en el cultivo de $L$. vannamei resultaron significativamente diferentes $\left(\mathrm{F}=4.99 \mathrm{E}^{+06}, \mathrm{p}<0.05\right)$ entre los tres tratamientos (Cuadro 2). El tratamiento EM1 presentó el mayor valor de ganancia diaria en peso $(0.0003 \pm 0.0001 \mathrm{~g} / \mathrm{d})$, TCE con $2.69 \pm 0.35 \mathrm{y}$ FCA con $1.46 \pm 0.20$. Los tratamientos C y EM2 no presentaron diferencias significativas entre sí (Tukey, $\mathrm{p}>0.05$ ), pero si fueron significativamente diferentes de EM1 en estos parámetros.

La supervivencia al final del ciclo de cultivo de L. vannamei para los tres tratamientos presentaron diferencias significativas $(\mathrm{F}=467.3, \mathrm{p}<0.05)$. El tratamiento $\mathrm{C}$ obtuvo la menor supervivencia con $45 \pm 2.00 \%$, mientras las mayores supervivencias se observaron con los tratamientos EM1 y EM2 con $61 \pm 8.76 \%$ y $60 \pm 10.5 \%$, respectivamente. La supervivencia de los camarones sin probióticos fue significativamente diferente (Tukey, $\mathrm{p}<0.05$ ) de los tratamientos EM1 y EM2, mientras que estos últimos no mostraron diferencias significativas entre sí (Tukey, $\mathrm{p}>0.05$ ).

Relaciones entre los parámetros de crecimiento y los parámetros ambientales: En el ACC se observó que la relación entre el peso y talla de los camarones y los parámetros ambientales en función de los tratamientos, pudo ser explicada por tres funciones canónicas (Cuadro 3). La primera función extraída explicó la máxima cantidad de varianza con $66.3 \%$, la cual indicó que los tratamientos estuvieron 
CUADRO 2

Efecto del probiótico en los parámetros de crecimiento de L. vannamei

TABLE 2

Probiotic effects on growth parameters of $L$. vannamei

\begin{tabular}{lccc}
\multicolumn{1}{c}{ Parámetros } & \multicolumn{3}{c}{ Tratamiento } \\
Ganancia en peso $(\mathrm{g})$ & $\mathrm{C}$ & EM1 & EM2 \\
Ganancia en peso $(\%)$ & $7.68 \pm 0.61^{\mathrm{a}}$ & $11.7 \pm 3.21^{\mathrm{a}}$ & $8.05 \pm 0.82^{\mathrm{a}}$ \\
Ganancia diaria en peso $(\mathrm{g} / \mathrm{d})$ & $511989 \pm 40851^{\mathrm{a}}$ & $593031 \pm 173062^{\mathrm{a}}$ & $407322 \pm 33707^{\mathrm{a}}$ \\
TCE $(\% / \mathrm{d})$ & $0.0001 \pm 0.0001^{\mathrm{b}}$ & $0.0003 \pm 0.0001^{\mathrm{a}}$ & $0.0002 \pm 0.00002^{\mathrm{b}}$ \\
FCA & $1.88 \pm 0.25^{\mathrm{b}}$ & $2.69 \pm 0.35^{\mathrm{a}}$ & $1.98 \pm 0.10^{\mathrm{b}}$ \\
$\mathrm{N}$ & $2.13 \pm 0.48^{\mathrm{a}}$ & $1.46 \pm 0.20^{\mathrm{b}}$ & $1.72 \pm 0.28^{\mathrm{a}}$ \\
\hline
\end{tabular}

Promedios en la misma fila con superíndices diferentes presentan diferencia significativa $(\mathrm{p}<0.05)$.

Promedio \pm desviación estándar.

$\mathrm{TCE}=$ Tasa de crecimiento específica. FCA=Factor de conversión alimenticia.

C: Control. EM1: Probiótico dosis 4L/ha. EM2: Probiótico dosis 10L/ha.
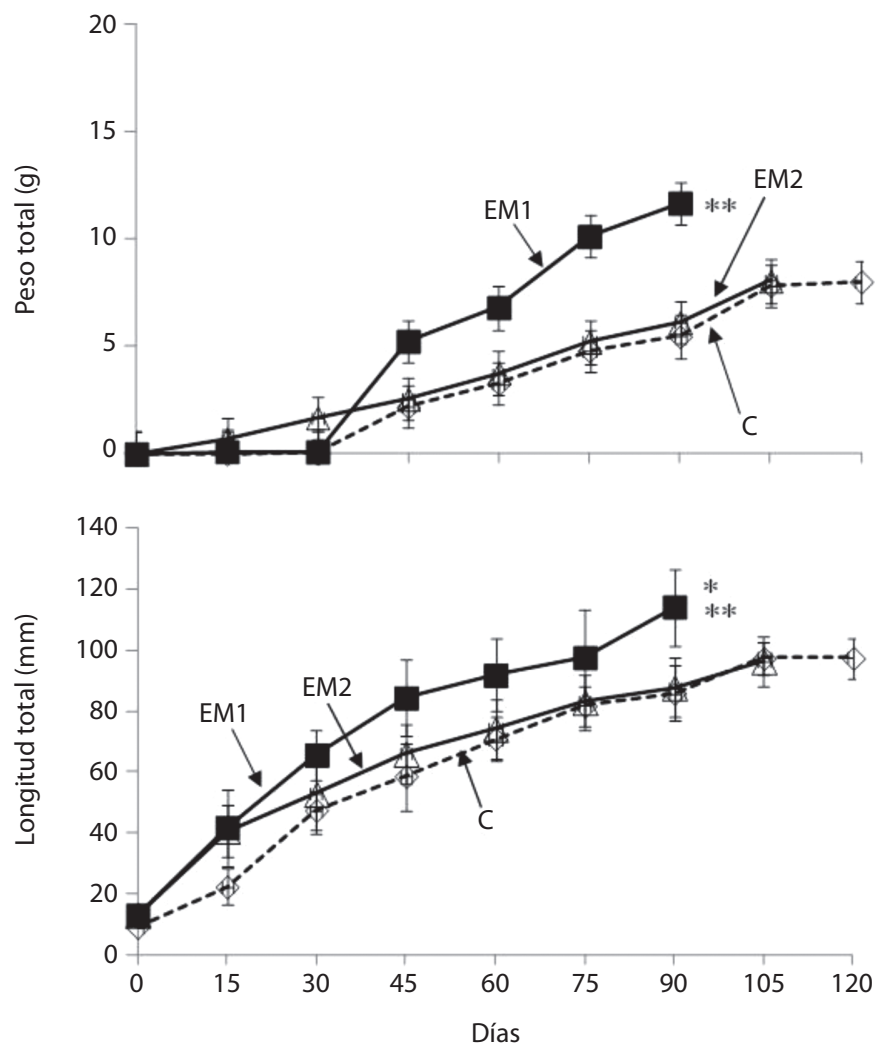

Fig. 2. Crecimiento en longitud y peso total de L. vannamei durante el período de cultivo en los diferentes tratamientos. C: Control. EM1: Probiótico dosis 4L/ha. EM2: Probiótico dosis 10L/ha. Los datos son expresados como promedio \pm desviación estándar. *Significativamente diferente respecto al control. **Significativamente diferente respecto al tratamiento EM2.

Fig. 2. Growth in total length and weight of L. vannamei during culture period in different treatments. C: Control. EM1: Doses probiotic 4L/ha. EM2: Doses probiotic 10L/ha. Data are expressed as mean \pm standard deviation. *Significantly different than control $(\mathrm{p}<0.05)$. **Significantly different than EM2 treatment. 
CUADRO 3

Análisis de correspondencia canónica entre los parámetros de crecimiento (peso y longitud total) y los parámetros ambientales en función de los tratamientos

TABLE 3

Canonic correspondence analysis between growth parameters (total length and weight) and the environmental parameters

\begin{tabular}{lcccc} 
& Eigenvalores & Inercia (\%) & $\begin{array}{c}\text { Factor de } \\
\text { Reductancia }\end{array}$ & $\begin{array}{c}\text { R canónico } \\
\text { (peso, longitud; ambientales) }\end{array}$ \\
Primera función canónica & 0.97 & 66.3 & 0.64 & 0.99 \\
Segunda función canónica & 0.56 & 47.5 & 0.26 & 0.75 \\
Tercera función canónica & 0.30 & 35.4 & 0.11 & 0.55 \\
\hline
\end{tabular}

asociados con el $\mathrm{pH}$ en agua, nitrógeno total y fósforo extraíble. Sin embargo, el $\mathrm{pH}$ en agua fue el parámetro que determinó la variabilidad en esta función con una correlación negativa, lo cual coincide con el AMD. La segunda función contribuyó con $47.5 \%$, observándose que las longitudes de los camarones se relacionaron con el $\mathrm{pH}$ en sedimento. Finalmente, la tercera función presentó la menor aportación de la varianza con $35.4 \%$, encontrándose que el oxígeno disuelto y la salinidad fueron determinantes en el peso de los camarones. No obstante, la salinidad fue el parámetro que representó la mayor variabilidad con una asociación negativa.

\section{DISCUSIÓN}

Efecto de los probióticos en los parámetros ambientales en el cultivo de camarón: Los probióticos en la acuicultura se han usado para evitar la aplicación de altas concentraciones de antibióticos y de diversos compuestos químicos, que pueden afectar la salud del consumidor, además de mejorar la calidad del agua y la supervivencia de los organismos cultivados (Balcázar et al. 2006, Villamil \& MartínezSilva 2009). Aunque la evaluación de los probióticos en la acuicultura se ha abordado en condiciones in vitro e in vivo desde diferentes líneas de investigación (nutrición, salud y calidad ambiental) (Günther \& Jiménez-Montealegre 2004, Chae-Woo 2009, Campa-Córdova 2011), la eficacia de estos productos comerciales disponibles en el mercado, continúa siendo poco conocida y controversial, debido a las condiciones no estandarizadas que se presentan en la producción a gran escala (Farzanfar 2006, Kesarcodi-Watson et al. 2008).

En el presente estudio, se observó que los parámetros de la calidad del agua se encontraron dentro de los intervalos recomendados para el cultivo intensivo de camarón $L$. vannamei con la inclusión de dos dosis del probiótico comercial (EM1 y EM2), a diferencia de los valores obtenidos en el tratamiento control (C), los cuales estuvieron fuera de los límites óptimos (Zhou et al. 2009). Es de esta forma que, las diferencias significativas observadas en la temperatura y la salinidad entre los tratamientos, muy probablemente estuvieron influenciadas por las condiciones ambientales presentadas durante el ciclo de producción (Ramírez-Rodríguez et al. 2006, Flores-Coto et al. 2009), y no necesariamente por el resultado de la actividad microbiana inducida por acción del producto.

En contraste, las diferencias observadas en los valores del $\mathrm{pH}$ mostraron que esta variable influyó en el comportamiento de los parámetros ambientales en función de los tratamientos determinándose una correlación negativa, es decir, que con el uso de la mezcla de $R$. palustris, $L$. plantarum, $L$. casei y $S$. cerevisiae en el cultivo de L. vannamei disminuyeron los valores del $\mathrm{pH}$. Estudios previos han reportado que los probióticos pueden producir diferentes compuestos orgánicos extracelulares como: quinonas, biotinas (bacterias fotosintéticas) (Farfanzar 2006, Qi et al. 2009), ácido láctico, 
peróxido de hidrógeno, acetaldehído, diacetilo, bacteriocinas (bacterias lácticas) (Rengpipat et al. 2008), vitaminas, glicerol, etanol, dióxido de carbono (Levaduras) (Folch-Mallol 2004), los cuales disminuyen el $\mathrm{pH}$ en el medio acuoso debido a sus características hidrofílicas (presencia de grupos hidroxilos) (Villamil \& Martínez-Silva 2009) como sucedió en la presente investigación. Lo anterior, concuerda con los trabajos realizados por Chae-Woo et al. (2009) y Banerjee et al. (2010), quienes reportaron que los valores del $\mathrm{pH}$ fueron regulados con el uso de bacterias lácticas y microalgas perifíticas para mejorar la calidad del agua en cultivos de camarones peneidos.

Por otra parte, algunos autores han mencionado la importancia del manejo del $\mathrm{pH}$ en los sistemas de cultivo, tanto en el agua como en los sedimentos, debido a la relación directa que tienen con la formación de compuestos tóxicos como: amonio, nitritos y nitratos (Boyd \& Tucker 1998, Cuéllar-Anjel 2010), los cuales pueden causar estrés, enfermedades y mortalidad en los camarones (Sansawat \& Thirabunyanon 2009, Ismail \& Soliman 2010). Aunque diversos estudios han mencionado que los probióticos no tienen efectos sobre la remoción de compuestos nitrogenados (Ajitha et al. 2004, Günther \& Jiménez-Montealegre 2004), los resultados presentados evidenciaron que los tratamientos EM1 y EM2 redujeron las concentraciones de $\mathrm{NO}_{3}-\mathrm{N}$, reconociéndose este último, como el mejor tratamiento, posiblemente causado por la dosis de aplicación, lo cual coincide con el valor del $\mathrm{pH}$ en estos mismos tratamientos.

Asimismo, las mayores concentraciones de oxígeno disuelto e incremento de la transparencia se registraron en los estanques adicionados con el producto comercial (tratamientos EM1 y EM2), lo cual sugiere que el crecimiento del fitoplancton puede controlarse con la adición de probióticos debido a la competencia por nutrientes y concuerda con los resultados reportados por Çetinkaya-Dönmez et al. (1999) y Kyum et al. (2004). Dichos autores mencionaron que existen algunas especies de bacterias fotosintéticas, (R. palustris) con la capacidad de fijar nitrógeno a partir de $\mathrm{NO}_{3}-\mathrm{N}$, y disminuir el florecimiento de las algas y por lo tanto, mantienen el equilibrio en la concentración de oxígeno disuelto. Igualmente, el $\mathrm{NO}_{3}-\mathrm{N}$ es un compuesto importante en el ciclo del nitrógeno y su reducción también puede deberse al proceso de desnitrificación que realizan bacterias autóctonas (Irianto \& Austin 2002).

Por otra parte, en el presente trabajo se demostró que al adicionar el producto comercial en los estanques de cultivo se redujo la concentración de NAT, lo cual concuerda con lo reportado por Banerjee et al. (2010), quienes al suministrar Bacillus pumilus y microalgas perifíticas en el agua en un cultivo controlado de $P$. monodon disminuyeron significativamente la concentración de NAT. Sin embargo, en nuestro caso únicamente, la reducción significativa en la concentración de este compuesto se vio reflejada en el tratamiento EM2 en comparación de los tratamientos C y EM1. Dicho comportamiento pudo estar asociado a que el tratamiento EM2 contuvo la mayor dosis de probiótico, lo cual indicó que su uso para este efecto fue correcto, aunque todos los tratamientos presentaron concentraciones dentro de los límites adecuados (0.1-1.0mg/L) para el cultivo de L. vannamei (Zhou et al. 2009). En contraste, McIntosh et al. (2000) probaron diferentes dosis de un probiótico comercial (Bacillus spp.) encontrándose efectos negativos (infección cutánea y menor tasa de supervivencia) en un cultivo intensivo de $L$. vannamei al incrementar la dosis de aplicación. En este sentido, diversos autores han mencionado que los probióticos pueden variar sus mecanismos de acción en respuesta a un ambiente controlado o en gran escala, así como en función de la dosis y concentración del consorcio microbiano (Balcazár 2006, Kesarcordi-Watson 2008, Villamil \& Martínez-Silva 2009). No obstante, Paéz-Osuna \& Frías-Espericueta (2001) mencionaron que la concentración de NAT puede depender de la interacción entre las variables: temperatura, alcalinidad, salinidad y $\mathrm{pH}$.

Con respecto a los cambios significativos observados en los niveles del agua en los tratamientos EM1 y EM2 en comparación con el 
tratamiento $\mathrm{C}$, se descartó que la profundidad haya sido un factor para determinar el efecto del probiótico, basándose en el análisis múltiple discriminante, el cual determinó que esta variable no fue significativa en el modelo, es decir, no posee una carga canónica considerable de la cual dependa el efecto del producto comercial. Sin embargo, la profundidad en los estanques de cultivo se encuentra dependiente del manejo técnico de la granja (Cuéllar-Anjel et al. 2010).

Los sistemas de cultivo intensivos se caracterizan por generar altos porcentajes de materia orgánica, los cuales se acumulan en el fondo del estanque como sedimento. En condiciones aerobias o anaerobias de descomposición, el material orgánico sedimentado se reincorpora a la columna de agua a través de minerales, compuestos químicos y gases (Torres-Beristarin 2005), que en exceso pueden ser tóxicos y causar la muerte para los organismos cultivados. Hasta el momento, son pocos trabajos que sugieren la aplicación de probióticos en la acuicultura con la finalidad de disminuir las cargas orgánicas en el sedimento (Avnimelech \& Ritvo 2003). En el presente estudio, se demostró que los tratamientos EM1 y EM2 redujeron significativamente los porcentajes de materia orgánica, mientras que los contenidos de fósforo extraíble se incrementaron. El aumento también se esperaba en los porcentajes de nitrógeno total en ambos tratamientos, sin embargo, el tratamiento EM2 presentó el mayor valor respecto al tratamiento C y EM1. Kumar et al. (2008) mencionaron que las bacterias probióticas juegan un papel importante en la degradación de la materia orgánica, lo que reduce significativamente su porcentaje en el sedimento y la formación de lodos, debido a que metabolizan rápidamente estos compuestos orgánicos convirtiéndolos en dióxido de carbono, minerales y biomasa celular. Aunque no existen intervalos recomendados para la calidad del sedimento en la acuicultura, Cuéllar-Anjel et al. (2010) establecieron que los porcentajes de materia orgánica se deben mantener bajos durante todo el ciclo de cultivo con la finalidad de evitar un ambiente anóxico en el fondo del estanque.

\section{Efecto de los probióticos en los paráme-} tros de crecimiento en el cultivo de camarón: El uso de probióticos en la acuicultura se ha asociado con un eficiente proceso de absorción y asimilación del alimento ingerido por parte de los organismos cultivados, debido a que estos microorganismos tienen la capacidad de colonizar el tracto gastrointestinal, en donde secretan nutrientes y enzimas digestivas que mejoran los procesos metabólicos y las respuestas inmunológicas de los hospederos (Günther \& Jiménez-Montealegre 2004, Balcázar et al. 2006).

La mayoría de las evidencias científicas indican que el mejor método de aplicación de los probióticos es a través del alimento con la finalidad de que las bacterias ingresen, colonicen y se multipliquen en el tracto digestivo (Irianto \& Austin 2002, Kumar et al. 2008). No obstante, en el presente estudio se demostró que con la adición de la mezcla de microorganismos eficientes en el agua del cultivo se contribuyó en la disminución de los días de cosecha (EM1 y EM2), en el incremento del peso y longitud final (EM1), en el aumento de la supervivencia (EM1 y EM2) y en el mejoramiento de la TCE y FCA (EM1). Alavandi et al. (2004) y Banerjee et al. (2010) encontraron que al adicionar bacterias lácticas en un cultivo de $P$. monodon mejoró el proceso de asimilación del alimento ingerido e incrementó la supervivencia de los camarones. Caso similar con lo reportado por Ismail \& Soliman (2010) quienes en un cultivo de Macrobrachium rosenbergii adicionaron al agua cepas de L. acidophilus, Streptococcus cremoris y L. bulgaricus obteniéndose una alta supervivencia. Sin embargo, McIntosh et al. (2000) aplicaron diferentes dosis de un consorcio comercial de microorganismos en un cultivo de L. vannamei observándose un efecto negativo en el proceso digestivo con el aumento de la dosis, pero sí mejoró la supervivencia.

Kesarcodi-Watson et al. (2008) mencionaron que los probióticos pueden mejorar el sistema inmunológico obteniéndose un efecto 
positivo en la supervivencia de los organismos cultivados en respuesta a un ambiente adverso. El tratamiento EM2 presentó parámetros fisicoquímicos dentro de los intervalos recomendados para el cultivo de L. vannamei (Díaz et al. 2001), sin embargo, la temperatura y la salinidad no estuvieron en las condiciones óptimas para el crecimiento de esta especie $\left(30^{\circ} \mathrm{C}\right.$ y 26 ups, respectivamente), lo que desde el punto de vista fisiológico la cantidad de energía adquirida por los camarones no se reflejó en el incremento en peso y longitud (Rosas et al. 1999), pero sí se obtuvo un mayor porcentaje de supervivencia. Es necesario considerar que los cambios hidro-meteorológicos son parte de las dificultades en el manejo operacional de los estanques, debido que la mayoría de las granjas de camarón se encuentran dentro de la línea costera (Cuéllar-Anjel et al. 2010).

Por otra parte, el ACC relacionó a la salinidad y el oxígeno con el peso, así como el pH del sedimento con la longitud de los camarones. Valdez et al. (2008) mencionaron la importancia de la salinidad y el consumo de oxígeno en las respuestas fisiológicas de peneidos, lo que se observa en un adecuado desarrollo y crecimiento. Sin embargo, estas variables se encuentran mayormente en función de los cambios ambientales y operacionales de la granja. Torres-Beristain (2005) argumentó que el crecimiento de los camarones es dependiente de la dinámica fisicoquímica en la interfase aguasedimento debido a que estos organismos habitan en el fondo del estanque. Además, Zhang et al. (2006) sugirieron que los cambios del pH interfieren en la muda de L. vannamei, mientras más alto se encuentre el valor, mayor es el tiempo que tarda en desprenderse el exoesqueleto. En este sentido, los valores del $\mathrm{pH}$ en el sedimento que se registraron en el tratamiento EM1 fueron moderadamente alcalinos, lo cual favorece el proceso de ecdisis.

Los resultados de este estudio demuestran una posible acción benéfica de los microorganismos eficientes incluidos en la mezcla comercial en las condiciones que se realizó esta investigación. Sin embargo, se debe de considerar que el efecto de su aplicación fue parcial debido a que sólo se pudieron constatar la regulación del $\mathrm{pH}$, disminución de la conversión alimenticia y reducción del tiempo de cosecha, que son algunos de los beneficios que los fabricantes ofrecen. Por otro lado, no se puede excluir los posibles efectos nocivos por la inclusión de bacterias exógenas y su relación entre las bacterias autóctonas no patógenas. Por lo tanto, se requieren estudios adicionales para determinar: 1) la dosis óptima de aplicación, 2) la dosis de emergencia en caso de cambios ambientales, 3) la colonización de los microorganismos eficientes en el tracto digestivo de los camarones, 4) si existe un efecto antagónico y 5) si hay un aumento de la respuesta inmune.

\section{AGRADECIMIENTOS}

A Jesús Mercado, Aarón Jarquín y Juan Juárez por su apoyo técnico, a la Fundación Produce Tabasco A. C. por el financiamiento otorgado para la realización de esta investigación a través del proyecto 27-2007-0415. A las personas responsables de la granja productora de camarón Aquatecnologías El Palmar por el apoyo brindado durante el desarrollo de este trabajo en campo.

\section{RESUMEN}

Los probióticos han ganado aceptación en la acuicultura para mantener la calidad del agua y aumentar el crecimiento de los organismos. En este estudio se analizó el efecto de una mezcla comercial de microorganismos eficientes (EM) (Rhodopseudomonas palustris, Lactobacillus plantarum, Lactobacillus casei y Saccharomyces cerevisiae) sobre la calidad del agua, sedimento y el crecimiento en un cultivo intensivo de camarón $L$. vannamei. La evaluación consistió en tres tratamientos: i) estanques sin EM (C), ii) estanques con dosis de 4L/ha (EM1) y iii) estanques con dosis de 10L/ha (EM2). Los resultados demostraron menor tiempo de cosecha en los tratamientos EM1 (90d) y EM2 (105d). Los tratamientos EM1 y EM2 mantuvieron significativamente regulados los valores del $\mathrm{pH}$ (EM1, 8.03 \pm 0.33 ; EM2, 7.77 \pm 0.22 ) y redujeron las concentraciones de nitrato (EM1, 0.64 $\pm 0.25 \mathrm{mg} / \mathrm{L}$; EM2, $0.39 \pm 0.26 \mathrm{mg} / \mathrm{L})$. El tratamiento EM2 presentó la mayor remoción de materia orgánica $(1.77 \pm 0.45 \%)$. El tratamiento EM1 mejoró la TCE $(2.69 \pm 0.35 \% / \mathrm{d})$ y FCA $(1.46 \pm 0.20)$. Los tratamientos EM1 y EM2 presentaron mayor supervivencia con $61 \pm 8.76 \%$ y $60 \pm 10.5 \%$, respectivamente. Este 
estudio demostró el efecto benéfico del uso de la mezcla comercial en los parámetros ambientales y de crecimiento en un cultivo intensivo de L. vannamei.

Palabras clave: acuicultura, calidad del agua, crecimiento, Litopenaeus vannamei, probióticos.

\section{REFERENCIAS}

Ajitha, S., M. Sridhar, N. Sridhar, I. Singh \& V. Varghese. 2004. Probiotic Effects of Lactic Acid Bacteria Against Vibrio Alginolyticus in Penaeus (Fenneropenaeus) indicus (H. Milne Edwards). Asian Fish. Sci. 17: 71-80.

Alavandi, S.V., K.K. Vijayan, T.C. Santiago, M. Poornima, K.P. Jithendran, S.A. Ali \& J.J.S. Rajan. 2004. Evaluation of Pseudomonas sp. PM11 and Vibrio fluviales PM17 on immune indices of tiger shrimp, Penaeus monodon. Fish Shellfish Immunol. 17: 115-120.

Anónimo. 2008. EMRO, Effective Microorganisms Research Organization. 2008. (Consultado: 22 febrero 2008, http://www.em-la.com/activacion_del_ emy1®.php?idioma=1).

Avnimelech, Y. \& G. Ritvo. 2003. Shrimp and fish pond soils: processes and management. Aquaculture 220: 549-567.

Balcázar, J.L., I. de Blas, I. Ruiz-Zarzuela, D. Cunningham, D. Vendrell \& J.L. Múzquiz. 2006. The rol of probiotics in aquaculture. Vet. Microbiol. 114: 173-186.

Balcázar, J.L., T. Rojas-Luna \& D.P. Cunningham. 2007. Effect of the addition of four potential probiotic strains on the survival of pacific white shrimp (Litopenaeus vannamei) following immersion challenge with Vibrio parahaemolyticus. J. Invertebr. Pathol. 96: 147-50.

Banerjee, S., H. Khatoon, M. Shariff \& F.M. Yusoff. 2010. Enhancement of Penaeus monodon shrimp postlarvae growth and survival without water exchange using marine Bacillus pumilus and periphytic microalgae. Fish. Sci. 76: 481-487.

Barón-Sevilla, B., L.F. Bückle-Ramírez \& M. HernándezRodríguez. 2004. Intensive culture of Litopenaeus vannamei BOONE 1931, in a recirculating seawater system. Ciencias Marinas 30: 179-188.

Boyd, C.E. \& C.S. Tucker.1998. Pond aquaculture water quality management. Kluwer Academic, Boston, Massachusetts, EE.UU.

Campa-Córdova, A.I., A. Luna-González, J.M. MazónSuastegui, G. Aguirre-Guzmán, F. Ascencio \& H.A. González-Ocampo. 2011. Efecto de bacterias probióticas en el cultivo larvario del ostión de placer Crassostrea corteziensis (Bivalvia: Ostreidae). Rev. Biol. Trop. 59: 183-191.

Çetinkaya-Dönmez, G., A. Öztürk \& L. Çakmakci. 1999. Properties of the Rhodopseudomonas palustris Strains Isolated From an Alkaline Lake in Turkey. Turk. J. Biol. 23: 457-463.

Chae-Woo, M., C. Yun-Seok \& O. Kye-Heon. 2009. Removal of pathogenic bacteria and nitrogens by Lactobacillus spp. JK-8 and JK-11. Aquaculture 287: 266-270.

Cuéllar-Anjel, J., C. Lara, V. Morales, A. De Gracia \& O. García Suárez. 2010. Manual de buenas prácticas de manejo para el cultivo del camarón blanco Penaeus vannamei. OIRSA-OSPESCA, Panamá.

Dalmin, G., K. Kathiresan \& A. Purushothaman. 2001. Effect of probiotics on bacterial population and health status of shrimp in culture pond ecosystem. Indian J. Exp. Biol. 39: 939-942.

Daniel, W. 2008. Bioestadística: Base para el análisis de las ciencias de la salud. Wiley, México, Distrito Federal, México.

Díaz, F., C. Farfán, E. Sierra \& A.D. Re. 2001. Effects of temperature and salinity fluctuation on the ammonium excretion and osmoregulation of juveniles of Penaeus vannamei, Boone. Mar. Freshwat. Behav. Physiol. 34: 93-104.

Douillet, P.A. 1998. Bacterial probiotic for water quality and disease control. World Aquaculture Society, Las Vegas, Nevada, EE.UU

Farzanfar, A. 2006. The use of probiotics in shrimp aquaculture. Immunol. Medical Microbiol. 48: 149-158.

Flores-Coto, C., M.L. Espinosa, F. Zavala \& L. Sanvicente. 2009. Ictioplancton del sur del Golfo de México. Un compendio. Hidrobiológica 19: 49-76.

Folch-Mallol, J.L., A. Garay-Arroyo, F. Lledías \& A. Covarrubias Robles. 2004. La respuesta a estrés en la levadura Saccharomyces cerevisiae. Rev. Latinoam. Microbiol. Parasitol. 46: 24-46.

Günther, J. \& R. Jiménez-Montealegre. 2004. Efecto del probiótico Bacillus subtilis sobre el crecimiento y alimentación de tilapia (Oreochromis niloticus) y langostino (Macrobrachium rosenbergii) en laboratorio. Rev. Biol. Trop. 52: 937-943.

Gutierrez-Wing, M.T. \& R. Malone. 2006. Biological filters in aquaculture: Trends and research directions for freshwater and marine applications. Aquac. Eng. 34: 163-171.

Irianto, A. \& B. Austin. 2002. Probiotics in aquaculture. J. Fish. Dis. 25: 633-642.

Ismail, M.M. \& W.S. Soliman. 2010. Studies on Probiotic Effects of Lactic Acid Bacteria Against Vibrio 
vulnificus in freshwater Prawn Macrobrachium rosenbergii. Am. J. Sci. 6: 781-787.

Jana, B.B. \& S. Jana. 2003. The potential and sustainability of aquaculture in India. J. Appl. Aquacult. 13: 283-316.

Kesarcodi-Watson, A., H. Kaspar, M.J. Lategan \& L. Gibson. 2008. Probiotics in aquaculture: The need, principles and mechanisms of action and screening processes. Aquaculture 274: 1-14.

Kristensen, E., S.I. Ahmed \& A.H. Deval. 1995. Aerobic and anaerobic decomposition of organic matter in marine sediment: which is fastest?. Limnol. Oceanogr. 40: 430-437.

Kumar, M., N.S. Swarnakumar, K. Sivakumar, T. Thangaradjou \& L. Kannan. 2008. Probiotics in aquaculture: importance and future perspectives. Indian J. Microbiol. 48: 299-308.

Kyum, M., K. Choi, C. Yin, K. Lee, I. Wan-Taek, J. Lim \& S. Lee. 2004. Odorous swine wastewater treatment by purple non sulfur bacteria, Rhodopseudomonas palustris, isolated from eutrophicated ponds. Biotechnol. Lett. 26: 819-822.

Ladino-Orjuela, G. \& J.A. Rodríguez-Pulido. 2009. Efecto de Lactobacillus casei, Saccharomyces cerevisiae, Rhodopeudomona palustris (microorganismos eficientes em) y melaza en la ganancia en peso de tilapias (Oreochromis sp.) en condiciones de laboratorio. Orinoquia 13: 31-36.

McIntosh, R.P., T.M. Samocha, E.R. Jones, A.L. Lawrence, D.A. McKee, S. Horowitz \& A. Horowitz. 2000. The effect of a commercial bacterial supplement on the high-density culturing of Litopenaeus vannamei with a low-protein diet in an outdoor tank system and no water exchange. Aquac. Eng. 21: 215-227.

Norma Oficial Mexicana (NOM-021-RECNAT-2000). Establece las especificaciones de fertilidad, salinidad y clasificación de suelo. Diario Oficial de la Federación. 31 diciembre 2002. México, Distrito Federal, México.

Paéz-Osuna, F. \& M.G. Frías-Espericueta. 2001. Toxicidad de los compuestos del nitrógeno en camarones, $\mathrm{p}$. 253-276. In F. Paéz-Osuna (ed.). Camaronicultura y Medio Ambiente. El Colegio de Sonora, Mazatlán, Sinaloa, México.

Panwichian, S., D. Kantachote, B. Wittayaweerasak \& M. Mallvarapuj. 2010. Isolation of purple nonsulfur bacteria for the removal of heavy metals and sodium from contaminated shrimp ponds. Electron. J. Biotechnol. (Consultado: 5 Abril 2011, DOI: 10.2225/ vol13-issue4-fulltext-8).

Qi, Z., X.H. Zhang, N. Boon \& P. Bossier. 2009. Probiotic in aquaculture of China-Current state, problems and prospect. Aquaculture 290: 15-21.
Ramírez-Rodríguez, M., F. Arreguín-Sánchez \& D. LluchBelda. 2006. Efecto de la temperatura superficial y la salinidad en el reclutamiento del camarón rosado Farfantepenaeus duorarum (Decapoda: Penaeidae), en la Sonda de Campeche, Golfo de México. Rev. Biol. Trop. 54: 1241-1245.

Rengpipat, S., T. Rueangruklikhit \& S. Piyatiratitivorakul. 2008. Evaluations of lactic acid bacteria as probiotics for juvenile seabass Lates calcarifer. Aquac. Res. 39: 134-143.

Rosas, C., L. Ocampo, G. Gaxiola, A. Sánchez \& L.A. Soto. 1999. Effect of salinity on survival, growth and oxygen consumption of postlarvae (PL10-PL21) of Penaeus setiferus. J. Crust. Biol. 19: 67-75.

Saad, A.S., M.M. Habashy \& K.M. Sharshar. 2009. Growth Response of the Freshwater Prawn, Macrobrachium rosenbergii (De Man), to Diets Having Different Levels of Biogen ${ }^{\circledR}$. World Appl. Sci. J. 6: 550-556.

Sansawat, A. \& M. Thirabunyanon. 2009. Anti-Aeromonas hydrophila activity and characterization of novel probiotic strains of Bacillus subtilis isolated from the gastrointestinal tract of giant freshwater prawns. J. Sci. Technol. 3: 77-87.

Shariff, M.F., M. Yusoff, T.N. Devaraja \& P.S. Srinivasa Rao. 2001. The effectiveness of a commercial microbial product in poorly prepared tiger shrimp, Penaeus monodon (Fabricius), ponds. Aquac. Res. 32: 181-187.

StatSoft. 2004. STATISTICA. Data analysis software system. Version 7. Tulsa, Oklahoma, EE.UU.

Ter Braak, C.J.F \& F.M. Verdonshot. 1995. Canonical correspondence analysis and related multivariate methods in Aquatic Ecology. Aquat. Sci. 57: 255-286.

Torres-Beristain, B. 2005. Organic matter descomposition in simulated aquaculture ponds. Ph.D. Thesis, Wageningen University, Wageningen, Netherlands.

Valdez, G., F. Díaz, A.D. Re \& E. Sierra. 2008. Efecto de la salinidad sobre la fisiología energética del camarón blanco Litopenaeus vannamei (Boone). Hidrobiológica 18: 105-115.

Venkat, H.K., N.P. Shau \& K.J. Jain. 2004. Effect on feeding Lactobacillus-based probiotics on the gut microflora, growth and survival of postlarvae of Macrobrachium rosenbergii (de Man). Aquac. Res. 35: 501-507.

Verschuere, L., G. Rombaut, P. Sorgeloos \& W. Verstraete. 2000. Probiotic bacteria as biological control agents in aquaculture. Microbiol. Mol. Biol. Rev. 64: 655-671.

Villamil, D.L. \& M.A. Martínez-Silva. 2009. Probióticos como herramienta biotecnológica en el cultivo de camarón: Reseña. Bol. Invemar 38: 165-187. 
Wang, Y.G., K.L. Lee, M. Najiah, M. Shariff \& M.D. Hassan. 2000. A new bacterial white spot syndrome (BWSS) in cultured tiger shrimp Penaeus monodon and its comparison with white spot syndrome (WSS) caused by virus. Dis. Aquat. Org. 41: 9-18.

Zar, J.H. 2010. Biostatistical Analysis. Prentice-Hall. Saddle River, New Jersey, EE.UU.

Zhang, P., X. Zhang, J. Li \& G. Huang. 2006. The effect of body weight, temperature, salinity, $\mathrm{pH}$, light intensity and feeding condition on lethal DO levels of whiteleg shrimp, Litopenaeus vannamei (Boone, 1931). Aquaculture 256: 579-587.

Zhou, X., Y. Wang \& W. Li. 2009. Effect of probiotic on larvae shrimp (Penaeus vannamei) based on water quality, survival rate and digestive enzyme activities. Aquaculture 287: 349-353.

Zokaei, F.H., C. Roos, H. Mohd, S. Azni \& S. Shakibazadeh. 2009. Effect of Bacillus subtilis on the growth and survival rate of shrimp (Litopenaeus vannamei). Afr. J. Biotechnol. 8: 3369-3376. 